Please do not remove this page

RMIT

UNIVERSITY

\title{
Influence of electromagnetic radiation on enzyme kinetics
}

Vojisavljevic, Vuk; Pirogova, Elena; Cosic, Irena

https://researchrepository.rmit.edu.au/esploro/outputs/9921863462001341/filesAndLinks?institution=61RMIT_INST\&index=null

Vojisavljevic, V., Pirogova, E., \& Cosic, I. (2007). Influence of electromagnetic radiation on enzyme kinetics. Proceedings of the 29th Annual International Conference of the IEEE EMBS, Lyon, France, 2007.

https://doi.org/10.1109/IEMBS.2007.4353468

Published Version: https://doi.org/10.1109/IEMBS.2007.4353468

Repository homepage: https://researchrepository.rmit.edu.au

(c) 2007 IEEE. Personal use of this material is permitted. However, permission to reprint/republish this material for advertising or promotional purposes or for creating new collective works for resale or redistribution to servers or lists, or to reuse any copyrighted component of this work in other works must be obtained from the IEEE.

Downloaded On 2023/04/26 21:19:03 +1000 


\title{
Influence of Electromagnetic Radiation on Enzyme Kinetics
}

\author{
V. Vojisavljevic, E. Pirogova, and I. Cosic, Senior Member, IEEE
}

\begin{abstract}
This study is focused on experimental validation of our hypothesis proposed within the Resonant Recognition Model (RRM) [7], [8] that protein function can be modified by an applied electromagnetic radiation of defined frequency in a range of infra red (IR), visible and ultra violet (UV) light. This postulate is investigated here by applying the electromagnetic radiation (1140-1200 $\mathrm{nm})$ to example of L-Lactate Dehydrogenase (LDH) protein and its biological activity is measured before and after the exposures. The presented methodology provides a possibility of enhancing the pharmaceutical and agricultural industries by amplifying drug potency via electromagnetic radiation.
\end{abstract}

\section{INTRODUCTION}

$\mathrm{L}$ ight activated changes in protein energy states have been shown to induce or modulate biological processes. For example light-activated excitation of the rhodopsin/bacteriorhodopsin molecules involved in the hyperpolarisation process of the cell membrane can either generate nerve impulses, ATP synthesis, or light regulated embryogenesis (i.e. seed germination in plants) [1]-[4]. It has also been suggested that Cytochrome c oxidase and certain dehydrogenases may play a key role in the photoreception process, particularly in the near infra-red (NIR) frequency range [4].

A number of different investigations [5], [6] have explored the effects of visible light on cell proliferation and metabolism. Studies of effects of low-intensity non-thermal light irradiation on eukaryotic and prokaryotic cells have reported positive results of accelerated proliferation rate in yeast and mammalian cells after irradiating with a $\mathrm{He}-\mathrm{Ne}$ laser [6] and increased E. coli proliferation during argon laser light exposure [4], [6]. An increased proliferation rate has also been observed in various bacterial cultures irradiated by laser light with radiant exposures at wavelengths of $630 \mathrm{~nm}$ and $810 \mathrm{~nm}$ [3].

The stimulating effect of various light-emitting diodes (LEDs) and monochromatic light on eukaryotic cells have been documented with pulsed and continuous light exposures. Several studies reported a change in

Manuscript received April 2, 2007. This work was proudly supported by the Australian Centre of Radiofrequency Bioeffects Research (ACRBR).

V. Vojisavljevic is with the School of Electrical and Computer Engineering, RMIT University, GPO Box 2476V Melbourne Victoria 3001 Australia (vojisavljevic@netscape.net).

E. Pirogova is with the School of Electrical and Computer Engineering, RMIT University, GPO Box 2476V Melbourne Victoria 3001 Australia (corresponding author: tel. 61-3-9925-3015, fax 61-3-9925-2007, elena.pirogova@rmit.edu.au)

I. Cosic is with the School of Electrical and Computer Engineering, RMIT University, GPO Box 2476V Melbourne Victoria 3001, Australia (email: irena.cosic@rmit.edu.au). acetylcholinesterase activity of human erythrocytes after low-intensity light radiation at $810 \mathrm{~nm}$. All these frequency selective effects of light on biological processes of protein activation imply that protein activation involves energies of the same order and nature as the electromagnetic irradiation of light [2]-[6].

All these processes can be observed in the light of the Resonant Recognition Model (RRM) which proposes that specificity of protein activations and interactions is electromagnetic in nature [7], [8] The RRM concept is based on the finding that there is a significant correlation between spectra of the numerical presentation of amino acid and their biological activity. It has been found through an extensive research that proteins with the same biological function have a common frequency in their numerical spectra. This frequency was found then to be a characteristic feature for protein biological function or interaction [7], [8].

In our previous study [9] we have tested the effects of visible light radiation in a range of 550-850 nm on enzyme kinetics of L-Lactate Dehydrogenase (LDH) enzyme. This paper presents the results of our further study of influence of EMR exposures on bioactivity of the $\mathrm{LDH}$ in the wavelengths ranging from 1140 up to $1200 \mathrm{~nm}$.

\section{MATERIALS AND METHODS}

\section{A. Computational analysis}

Each biological process involves a number of interactions between proteins and their targets, which are based on the energy transfer between the interacting molecules. Protein interactions are highly selective, and this selectivity is defined within the protein structure. The RRM is designed for analysis of protein (DNA) interactions and their interaction with EMR [7]-[13].

The application of the RRM involves two stages of calculation. The first is the transformation of the amino acid sequence into a numerical sequence. Each amino acid is represented by its Electron-Ion Interaction Potential (EIIP) value which describes the average energy states of all valence electrons in a given amino acid [14]. A unique number can thus represent each amino acid or nucleotide, irrespective of its position in a sequence. Then the numerical series obtained are analyzed by digital signal analysis methods, Fourier and Wavelet transform, in order to extract information pertinent to the biological function. A multiple cross-spectral function is defined and calculated to obtain the common frequency components from the spectra of a group of proteins. Peaks in such function denote common frequency components for all sequences analyzed. Peak 
frequencies in such a multiple cross-spectral function denote common frequency components for all sequences analyzed.

In our previous work [7]-[13] a relationship between the RRM spectra of some protein groups and their interaction with visible light was established. It has been shown that all protein sequences with a common biological function have a common frequency component in the free energy distribution of electrons along the protein backbone. This characteristic frequency was shown to be related to protein biological function [7]-[13]. Furthermore, it was also shown that proteins and their targets share a characteristic frequency. Thus, it can be further postulated that RRM frequencies characterize not only a general function but also a recognition/interaction between the particular proteins and their target at a distance. Thus, protein interactions can be viewed as a resonant energy transfer between the interacting molecules. This energy can be transferred through oscillations of a physical field, possibly electromagnetic in nature [7]-[13].

Since there is evidence that proteins have certain conducting or semi-conducting properties, a charge moving through the protein backbone and passing different energy stages caused by different amino acid side groups can produce sufficient conditions for a specific electromagnetic radiation or absorption. In our previous research we have shown that such charge transfer through the protein backbone is possible through an exciton process [7].

A strong linear correlation exists between the predicted and experimentally determined frequencies corresponding to the absorption of electromagnetic radiation of such proteins [7], [9]. It is inferred that approximate wavelengths in real frequency space can be calculated from the RRM characteristic frequencies for each biologically related group of sequences. These calculations can be used to predict the wavelength of the light irradiation, which might affect the biological activity of exposed proteins [7], [9]. The frequency range predicted for protein interactions is from $10^{13} \mathrm{~Hz}$ to $10^{15} \mathrm{~Hz}$. This estimated range includes IR, visible and UV light. These computational predictions were confirmed by comparison of:

a) Absorption characteristics of light absorbing proteins and their characteristic RRM frequencies [7], [11].

b) Frequency selective light effects on cell growth and characteristic RRM frequencies of growth factors [7], [12].

c) Activation of enzymes by laser radiation [7], [9], [13].

All these results indicate that the specificity of protein interaction is based on a resonant electromagnetic energy transfer at the frequency specific for each interaction observed. A linear correlation between the absorption spectra of proteins and their RRM spectra with a regression coefficient of $\mathrm{K}=201$ has been established. Using RRM postulates, a computationally identified characteristic frequency for a protein functional group can be used to calculate the wavelength of applied irradiation, $\lambda$, which assumingly would activate this protein sequence and modify its bioactivity [7]:

$$
\lambda=201 / f_{\text {RRM }}
$$

Here we will utilize this relationship to calculate the frequencies/wavelengths that might modulate the bioactivity of the selected enzymes and investigate/evaluate their activation experimentally.

\section{B. Experimental study}

Enzymes are proteins crucial in accelerating metabolic reactions in the living organism. Dehydrogenases are the enzymes that catalyze a variety of oxidation-reduction reactions within the cells. As the protein example we have chosen the L-Lactate Dehydrogenase (rabbit muscle). This enzyme has been selected on the basis its commercial availability, simplicity of the assay, and the possibility of measuring their bioactivity using the standard well accepted procedure, i.e. Continuous Spectrophotometric Rate Determination. As a source of IR and visible light we have used a SpectraPro 2300i monochromator (Acton Research Corporation) with a wavelength range of 400-1200 nm, grating $600 \mathrm{~g} / \mathrm{mm}$ and a resolution of $0.1 \mathrm{~nm}$. For measurement of absorbance of the analyzed enzyme solutions we will use an Ocean Optics USB2000 spectrometer coupled to a CCD array which can detect in the 190-870 nm range. Software control automatically monitors and saves the optical activity values at the required wavelength every $30 \mathrm{sec}$.

L-lactate dehydrogenase (LDH) rabbit muscle EC1.1.1.27 catalyses the inter-conversion of the 1-lactate into pyruvate with the Nicotinamide Adenine Dinucleotide Oxidised form (NAD+) acting as a coenzyme. The suitability of the LDH enzyme for this reaction is attributed to the absorption characteristics of the NADH (Nicotinamide Adenine Dinucleotide Reduced form). NADH is able to absorb light at $340 \mathrm{~nm}$ in contradict to the NAD, which is inactive at this frequency. Due to the different optical characteristics of the $\mathrm{NADH}$ and NAD we are able to optically asses if the reaction Pyruvate $\rightarrow$ Lactate in the presence of the LDH as an accelerator has occurred and then determine the amount of the reactants. The experimental procedure is presented below:

1. The samples are irradiated for $10 \mathrm{~min}$ using Monochromator SpectraPro 2150i (ACTON RESEARCH CORPORATION) set at the activation wavelength identified computationally using the RRM approach.

2. These irradiated samples are added to the already prepared solution of NADH and pyruvate.

3. The optical density of NADH are measured at $340 \mathrm{~nm}$.

4. The values of rate of change in absorbance of NADH and changes of absorption coefficient values (at $340 \mathrm{~nm}$ ) in time are collected and presented graphically.

\section{RESULTS AND DISCUSSION}

In our previous work [9] we have analyzed thirty two eukaryotic dehydrogenase sequences using the RRM approach. A multiple cross-spectral analysis was performed resulting in two characteristic frequencies identified at 
$\mathbf{f}_{\mathbf{1}}=\mathbf{0 . 1 6 8 8} \pm \mathbf{0 . 0 0 4}$ and less prominent at $\mathbf{f}_{2}=\mathbf{0 . 2 3 9 2} \pm \mathbf{0 . 0 0 4}$ (Fig.1). These frequencies are related to the biological activity as it was found in our previous investigations [9]. Based on the characteristic frequencies determined for the whole dehydrogenase functional group, we have calculated the wavelength of irradiation, $\lambda$, which assumingly would activate dehydrogenase sequences and modify their bioactivity:

$$
\lambda=201 / f_{\text {RRM }}
$$

Thus, the wavelengths of the electromagnetic exposure required for dehydrogenase enzymes activation would be at $1191 \pm 15 \mathrm{~nm}$ and $846+15 \mathrm{~nm}$. In our previous study we investigated the effects of EMR $(550-850 \mathrm{~nm})$ on the LDH kinetics [9]. Here we have studied the changes in the LDH activity upon irradiation in a range of 1140-1200 nm.

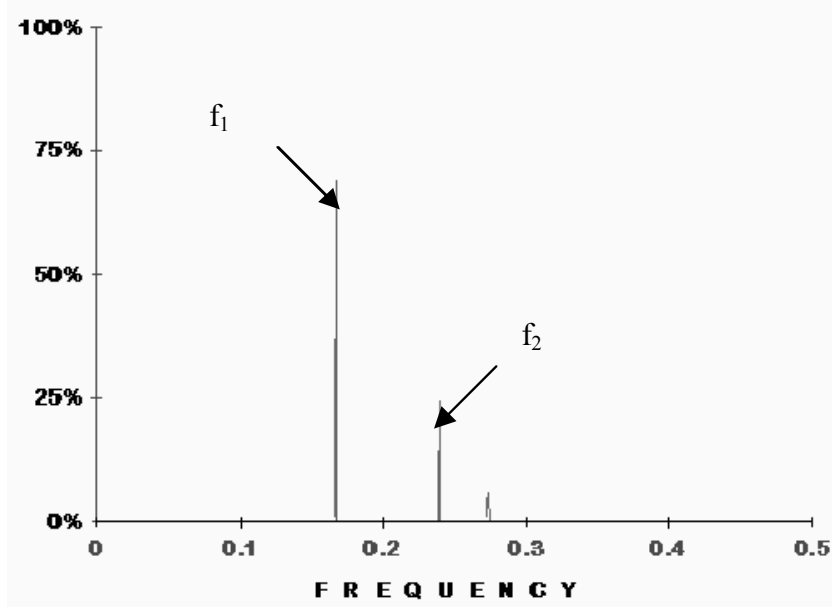

Fig. 1 Multiple cross-spectral function of Dehydrogenase proteins (32 sequences). The prominent peak(s) denote common frequency components. The abscissa represents RRM frequencies, and the ordinate is the normalized intensity.

\section{A. Measurement of the absorption spectrum of NADH}

1. We have diluted the stock coenzyme solution with the $0.003 \mathrm{M}$ potassium phosphate assay buffer.

2. Using the properly diluted coenzyme solution, we have measured the absorbance at 2-5 nm intervals between 1140 and $1200 \mathrm{~nm}$.

3. The spectrophotometer is set to $100 \%$ transmittance (zero absorbance) at each wavelength using the $0.003 \mathrm{M}$ $\mathrm{K}_{2} \mathrm{HPO}_{4}$ assay buffer blank.

The results obtained have shown that NADH concentration corresponds to the maximum absorbance of 1.6 at $340 \mathrm{~nm}$. Fig. 2 shows how NADH sample absorbance is affected by the applied radiation of the defined wavelength.

\section{B. Measurement of LDH activity}

$2.5 \mathrm{ml}$ cuvettes are filled with the following components: $0.1 \mathrm{ml}$ of $0.0027 \mathrm{M}$ Sodium Pyruvate (BioWhittaker);

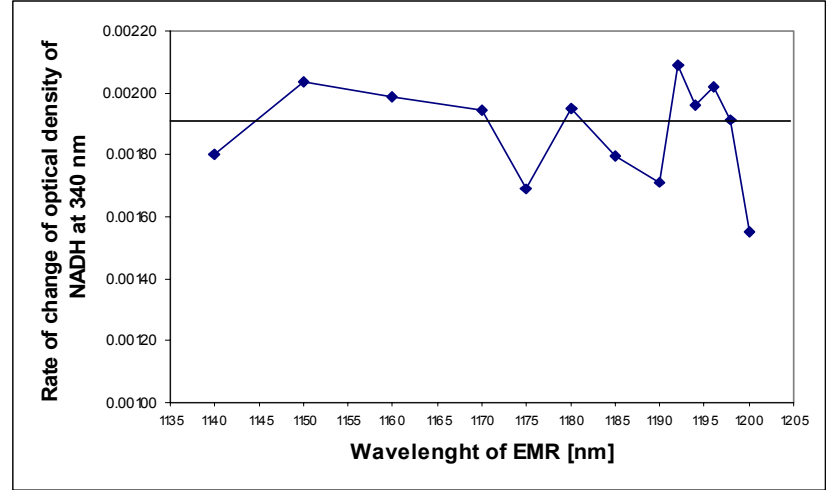

Fig. 2 Gradient of change in absorbance of NADH

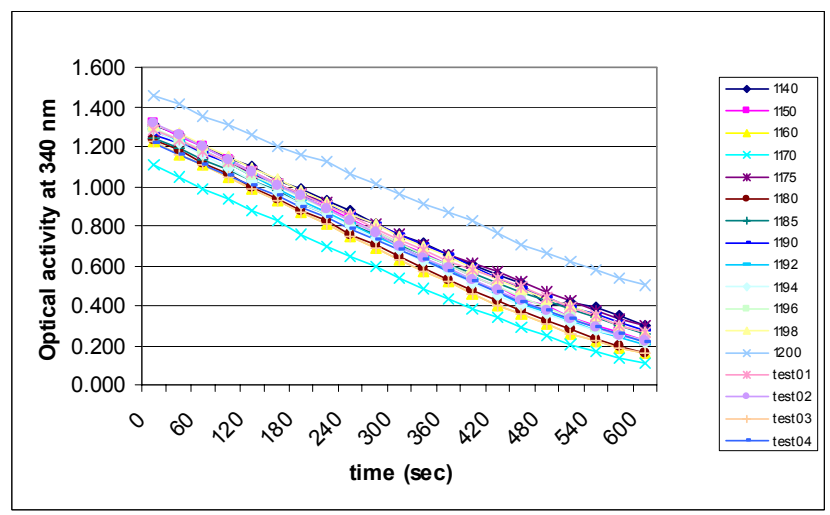

Fig. 3 Changes of absorption coefficient values (at $340 \mathrm{~nm}$ ) in time after irradiation with different wavelengths

$0.1 \mathrm{ml} \mathrm{NADH}$; disodium salt $(\mathrm{C} 21 \mathrm{H} 27 \mathrm{~N} 7 \mathrm{O} 14 \mathrm{P} 2 \mathrm{Na} 2$ Roche); 0.005M Phosphate buffered saline (SIGMA); $1.5 \mathrm{ml}$ of deionized water; $0.3 \mathrm{ml} \mathrm{LDH}$ diluted in $2 . \mathrm{g} / \mathrm{ml}$ of Phosphate buffered saline with BSA (SIGMA).

The experiments were performed at room temperature $27^{\circ} \mathrm{C}$ (Temperature controller Quantum Northwest). The cuvettes were filled with $0.3 \mathrm{ml}$ of the $\mathrm{LDH}$ samples. The samples were previously irradiated with the light of different wavelengths $(1140-1200 \mathrm{~nm})$ for $600 \mathrm{sec}$. These irradiated samples were added to the already prepared solution of NADH and pyruvate. The optical density of NADH was measured at $340 \mathrm{~nm}$ foe each irradiating wavelength of light and is presented in Table 1 and Fig. 3.

The results obtained have shown the change of absorbance of NADH samples under the influence of irradiated LDH. After radiation in the range of 1140-1200 $\mathrm{nm}$, the LDH bioactivity has increased resulting in accelerating the reaction Pyruvate + NADH ------> Lactate + $\mathrm{NAD}^{+}+\mathrm{H}^{+}$. From Fig.2 we can observe that maximum optical density of NADH is achieved at the wavelengths 1192 and $1200 \mathrm{~nm}\left(\mathrm{f}_{1}=0.1688 \pm 0.004\right)$ as was predicted by the RRM as the possible activation frequency of dehydrogenase enzymes. Hence, the results suggest that this specific biological process can be modulated by irradiation with defined frequencies strongly supporting the main concept of the RRM methodology. The possibility to computationally calculate the frequencies with the following use of IR and visible light to produce the desired biological 
mutations and alterations in proteins would benefit the development of new biomaterials, non-invasive treatments and advanced technologies.

\section{CONCLUSION}

All these results lead to the conclusion that the specificity of protein interactions is based on the resonant electromagnetic energy transfer at the frequency specific for each interaction can be predicted using the RRM Model. Furthermore, it has been shown that proteins activity can be modulated by electromagnetic radiation at the specific frequency as predicted by the RRM. Thus, based on the RRM spectral characteristic, it is possible to calculate the wavelength of electromagnetic energy that can be used to modulate the protein activity hence giving rise to an innovative methodology to program, predict, design and modify proteins and their bioactivity. This could have major implications in drug design, medicine, agriculture, pharmacology and biotechnology.

\section{REFERENCES}

[1] E. J. Lerner .--ed., "Biological effects of electromagnetic fields", IEEE Spectrum, 1984.

[2] T. Karu, "Photobiological fundamentals of low-power laser therapy", IEEE Journal of Quantum Electronics, QE-23, pp. 1703-1717, 1987.
[3] T. Karu, "Primary and Secondary Mechanisms of Actions of Visible to Near-IR Radiation on Cells", J. Photochem. Photobiol, Vol. 49, pp.1-17, 1999.

[4] Blank M, Soo L. 2001. Optimal frequencies for magnetic acceleration of cutochrome oxidase and NaK-ATPase reactions. Bioelectrochemistry and Bioenergetics. V. 53(2): 171-174.

[5] M. Ahma, A.R. Cashmore, "HY4 Gene of A. Thaliana Encodes a Protein with Characteristics of Blue-light Photoreceptor", Nature, Vol. 366, pp. 162-166, 1993.

[6] H. Blum, Carcinogenesis by Ultraviolet Light, (N.J.), Princeton University Press, Princeton, 1959.

[7] I. Cosic, The Resonant Recognition Model of Macromolecular Bioactivity: Theory and Applications, Basel, Birkhauser Verlag, 1997.

[8] I. Cosic, "Macromolecular Bioactivity: Is it Resonant Interaction between Macromolecules?-Theory and Applications", IEEE Trans. on Biomedical Engineering, Vol. 41, pp. 1101-1114, 1994.

[9] V. Vojisavljevic, E. Pirogova, I. Cosic, "The effect of Electromagnetic Radiation (550nm-850nm) on 1-Lactate Dehydrogenase Kinetics", Radiation Biology (accepted for publication 18 September 2006).

[10] I. Cosic, "Virtual Spectroscopy for Fun and Profit", Biotechnology, Vol. 13, pp. 236-238, 1995.

[11] I. Cosic, S. Birch, "Photoreceptors Having Similar Structure but Different Absorptions Can be Distinguished using the Resonant Recognition Model", Proc. IEEE EMBS, Vol. 16, pp. 265-266, 1994.

[12] I. Cosic, V. Vojisavljevic, M. Pavlovic, "The Relationship of the Resonant Recognition Model to effects of Low-intensity Light on Cell Growth", Int. J. Radiat. Biology, Vol. 56, pp. 179-191, 1989.

[13] G. Biscar, "Photon Enzyme Activation", Bull. Math. Biology, Vol. 38, pp.:29-38, 1986.

[14] Veljkovic V, Slavic I. 1972. General model of pseudopotentials, Physical Review Letters. 29, 105-108.

TABLE 1.

ABSORBANCE AND WAVELENGTH VALUES OF NADH SAMPLE

\begin{tabular}{|c|c|c|c|c|c|c|c|c|c|c|c|c|c|c|}
\hline \multirow[b]{2}{*}{$\mathrm{t}[\mathrm{sec}]$} & \multicolumn{14}{|c|}{ Wavelength of irradiating light [nm] } \\
\hline & 1140 & 1150 & 1160 & 1170 & 1175 & 1180 & 1185 & 1190 & 1192 & 1194 & 1196 & 1198 & 1200 & test 01 \\
\hline 0 & 1.311 & 1.318 & 1.223 & 1.104 & 1.276 & 1.235 & 1.243 & 1.261 & 1.286 & 1.285 & 1.296 & 1.307 & 1.456 & 1.283 \\
\hline 30 & 1.257 & 1.255 & 1.161 & 1.048 & 1.231 & 1.186 & 1.191 & 1.218 & 1.228 & 1.217 & 1.233 & 1.268 & 1.411 & 1.238 \\
\hline 60 & 1.196 & 1.196 & 1.104 & 0.989 & 1.169 & 1.115 & 1.132 & 1.168 & 1.184 & 1.153 & 1.185 & 1.205 & 1.353 & 1.176 \\
\hline 90 & 1.138 & 1.131 & 1.049 & 0.938 & 1.121 & 1.061 & 1.082 & 1.117 & 1.113 & 1.089 & 1.116 & 1.146 & 1.307 & 1.125 \\
\hline 120 & 1.096 & 1.071 & 0.986 & 0.878 & 1.067 & 1.000 & 1.029 & 1.058 & 1.053 & 1.028 & 1.055 & 1.088 & 1.260 & 1.074 \\
\hline 150 & 1.031 & 1.010 & 0.931 & 0.824 & 1.016 & 0.936 & 0.975 & 1.012 & 0.987 & 0.970 & 1.004 & 1.037 & 1.201 & 1.014 \\
\hline 180 & 0.983 & 0.947 & 0.866 & 0.758 & 0.962 & 0.877 & 0.913 & 0.956 & 0.927 & 0.914 & 0.942 & 0.978 & 1.158 & 0.960 \\
\hline 210 & 0.926 & 0.891 & 0.806 & 0.696 & 0.915 & 0.823 & 0.863 & 0.912 & 0.871 & 0.859 & 0.882 & 0.918 & 1.120 & 0.904 \\
\hline 240 & 0.873 & 0.831 & 0.752 & 0.649 & 0.853 & 0.755 & 0.811 & 0.858 & 0.811 & 0.797 & 0.827 & 0.859 & 1.066 & 0.850 \\
\hline 270 & 0.813 & 0.772 & 0.689 & 0.596 & 0.808 & 0.703 & 0.762 & 0.805 & 0.751 & 0.741 & 0.771 & 0.810 & 1.011 & 0.787 \\
\hline 300 & 0.761 & 0.714 & 0.629 & 0.538 & 0.754 & 0.644 & 0.706 & 0.755 & 0.694 & 0.681 & 0.712 & 0.752 & 0.961 & 0.732 \\
\hline 330 & 0.711 & 0.661 & 0.572 & 0.488 & 0.704 & 0.589 & 0.659 & 0.705 & 0.636 & 0.622 & 0.657 & 0.695 & 0.913 & 0.678 \\
\hline 360 & 0.656 & 0.608 & 0.515 & 0.436 & 0.656 & 0.530 & 0.603 & 0.653 & 0.579 & 0.571 & 0.606 & 0.643 & 0.868 & 0.625 \\
\hline 390 & 0.601 & 0.547 & 0.458 & 0.385 & 0.613 & 0.480 & 0.561 & 0.596 & 0.524 & 0.512 & 0.545 & 0.583 & 0.823 & 0.575 \\
\hline 420 & 0.550 & 0.494 & 0.404 & 0.336 & 0.566 & 0.429 & 0.513 & 0.535 & 0.469 & 0.455 & 0.495 & 0.530 & 0.769 & 0.537 \\
\hline 450 & 0.509 & 0.435 & 0.354 & 0.290 & 0.518 & 0.374 & 0.464 & 0.488 & 0.413 & 0.404 & 0.442 & 0.482 & 0.708 & 0.496 \\
\hline 480 & 0.403 & 0.403 & 0.304 & 0.244 & 0.470 & 0.324 & 0.426 & 0.444 & 0.366 & 0.358 & 0.392 & 0.435 & 0.666 & 0.440 \\
\hline 510 & 0.420 & 0.341 & 0.259 & 0.205 & 0.427 & 0.279 & 0.379 & 0.394 & 0.319 & 0.314 & 0.340 & 0.396 & 0.620 & 0.387 \\
\hline 540 & 0.392 & 0.300 & 0.221 & 0.170 & 0.373 & 0.234 & 0.338 & 0.358 & 0.276 & 0.276 & 0.292 & 0.346 & 0.576 & 0.348 \\
\hline 570 & 0.348 & 0.260 & 0.189 & 0.137 & 0.335 & 0.198 & 0.302 & 0.314 & 0.238 & 0.243 & 0.255 & 0.300 & 0.538 & 0.301 \\
\hline 600 & 0.301 & 0.225 & 0.160 & 0.110 & 0.294 & 0.162 & 0.259 & 0.275 & 0.207 & 0.211 & 0.223 & 0.267 & 0.498 & 0.267 \\
\hline Grad. & 0.0018 & 0.0020 & 0.0020 & 0.0019 & 0.0017 & 0.0020 & 0.0018 & 0.0017 & 0.0021 & 0.0020 & 0.0020 & 0.0019 & 0.0016 & 0.0018 \\
\hline
\end{tabular}

